Revisiones de literatura

\title{
Análisis bibliométrico y de contenido a la producción científica de la psicología con relación al estudio del emprendimiento: hacia una nueva propuesta conceptual
}

\author{
Bibliometric and content analysis of the scientific production of psychology relat- \\ ed to the study of entrepreneurship: towards a new conceptual proposal
}

\author{
Quezada, Mirna Elizabeth ${ }^{1}$, Vega Valero, Cynthia Zaira ${ }^{1}$ y Nava Quiroz, Carlos ${ }^{1}$
}

\begin{abstract}
Resumen:
El emprendimiento ha tenido un reciente auge de estudio por varias las disciplinas que se han preocupado por el fenómeno, entre éstas la psicología. El objetivo del presente estudio fue analizar el vasto trabajo de la psicología bajo un enfoque sistemático de la literatura, con el fin de identificar lagunas en la investigación del emprendimiento por parte de la disciplina en las que se pudiera insertar una nueva propuesta conceptual. Se realizó un análisis bibliométrico a 532 textos científicos resultado de criterios de inclusión y exclusión y se obtuvieron los principales países, universidades y autores que han contribuido a la temática. Se realizó también un análisis de contenido inductivo de los textos más citados de la muestra anterior $(n=15)$ con el fin de obtener un mapa de líneas de investigación el cual se confirmó y detalló con un análisis de contenido deductivo a los textos científicos con el $80 \%$ de citas acumuladas $(n=100)$. Resultado de esto se contempla la posibilidad de incluir el estudio del emprendimiento desde el comportamiento emprendedor.
\end{abstract}

Palabras Clave: emprendimiento, revisión sistemática, análisis bibliométrico, análisis de contenido, comportamiento emprendedor.

${ }^{1}$ Universidad Nacional Autónoma de México

*Correspondencia: psc.m.quezada@gmail.com

\begin{abstract}
:
Entrepreneurship has had a recent study boom by various disciplines that have been concerned about the phenomenon, among these is psychology. The aim of this paper was to analyze the work of psychology under a systematic approach to the literature, in order to identify gaps by the discipline in the research of entrepreneurship in which a new conceptual proposal could be inserted. A bibliometric analysis was carried out on 532 scientific texts resulting from inclusion and exclusion criteria, and the main countries, universities and authors who have contributed to the subject were obtained. An inductive content analysis of the most cited texts of the previous sample $(n=15)$ was also carried out in order to obtain a map of research lines which was confirmed and detailed with a deductive content analysis of the scientific texts with $80 \%$ of accumulated citations $(\mathrm{n}=100)$. As a result of this, the possibility of including the study of entrepreneurship from entrepreneurial behavior is contemplated.
\end{abstract}

Keywords:entrepreneurship, systematic review, bibliometric analysis, content analysis, entrepreneurial behavior. 
El emprendimiento ha tenido reciente auge de estudio por una vasta red de interesados en un crecimiento económico a partir de la competencia que involucra un mundo globalizado (Tangarife, Giraldo y Sanín, 2015; Santos, Barrozo y Guzmán, 2013). Las naciones se han preocupado por potenciar en su población el surgimiento de emprendedores que activen y mantengan latente la economía local, con el propósito de hacer frente a los retos económicos y sociales actuales (Minniti, 2012).

La comunidad científica no se ha quedado atrás y se ha encaminado a la comprensión del fenómeno desde distintos enfoques propios de la disciplina que representan, como lo es la economía, sociología, administración, así como la psicología, entre otras, todas interesadas en la comprensión del fenómeno para generar una red de conocimiento que permita la manipulación de los factores que inciden en éste (Low y MacMillan, 2007).

La primera disciplina que tuvo interés en el emprendimiento fue la economía; en Francia desde el siglo XVIII se denominó entrepreneur a la persona que intercambiaba algún bien o servicio con fines de lucro (Najaragan, 2011). Desde ese momento y con el paso del tiempo se comenzaron a delimitar la serie de características de los emprendedores que promovían el crecimiento económico con su acción emprendedora.

Es hasta mediados del siglo XX con los trabajos del psicólogo estadounidense David McClelland que la psicología enfatiza su interés por el fenómeno, enfocando sus esfuerzos al estudio de las características psicológicas que permitían distinguir a un emprendedor del resto de la sociedad (Carlsson et al., 2013). Tal ha sido el aporte de la psicología al estudio del fenómeno, que sólo después de las ciencias de la administración es la disciplina más fructífera en cuanto a investigación dedicada a emprendimiento (Ireland y Webb,
2007); por tal motivo el psicólogo interesado en investigar al emprendedor y su acción emprendedora requiere de manera puntual indagar en el vasto cúmulo de producción científica que se ha generado en los últimos años, a fin de identificar con claridad las preguntas no resueltas por la disciplina hasta el momento.

Por tal motivo, algunos autores se han orientado a delimitar cuales han sido las contribuciones específicas de la disciplina al campo del emprendimiento. Por ejemplo, Rosero y Molina (2008) ofrecen una clasificación para revisar el trabajo de investigación que ha desarrollado la psicología desde mediados del siglo $\mathrm{XX}$; los autores mencionan que las investigaciones se pueden clasificar en tres tendencias principalmente: a) los trabajos orientados a los rasgos psicológicos del emprendedor; b) las investigaciones centradas en el proceso de desarrollo del emprendimiento; y c) las aportaciones dirigidas al estudio de factores cognitivos relacionados con el aprendizaje del emprendedor.

De igual manera, Veciana (2007) hace una revisión y menciona que es posible distinguir dos tipos de aportaciones de la psicología al emprendimiento, un nivel macro y micro; el primer conjunto de aportaciones lo denomina enfoque psicológico micro, dentro de esta clasificación se pueden encontrar investigaciones sobre los rasgos de personalidad que distinguen a los emprendedores; la segunda clasificación desde un enfoque psicológico macro, la define como comportamiento del empresario orientado a la gerencia, en la cual se concentran estudios dirigidos a identificar, describir y explicar el comportamiento del empresario.

Sin embargo, tanto Rosero y Molina (2008) como Veciana (2007), realizaron el análisis con base en revisiones narrativas de la literatura, las cuales son más susceptibles a 
imprecisiones y sesgos en la identificación, evaluación, resumen y análisis de la literatura científica en comparación con las revisiones sistemáticas (Lozano, 2005; González, Urrutia y Alonso-Coello, 2011; Beltrán, 2005).

En este sentido, para poder examinar el aporte de la psicología al estudio del emprendimiento, es requerido realizar un análisis de la literatura que permita explorar de manera sistemática entre la diversa producción científica del tema, y de esta forma describir el conocimiento existente de manera precisa a fin de generar hipótesis, preguntas de investigación o delimitar ámbitos de estudio que no se han trabajado hasta el momento; dicho análisis se denomina revisión sistemática exploratoria o scooping review, y es mejor conocido como análisis bibliométrico (Poth y Ross, 2009; Manchado-Garabitio et al., 2009).

El análisis bibliométrico es un método de revisión sistemática que consiste en desarrollar cuantitativamente el cuerpo de conocimiento de una disciplina, esto se logra a través de la identificación de las tendencias en producción científica en determinado periodo de tiempo y su impacto en la comunidad científica, entendiendo el impacto como la identificación de las contribuciones con mayor influencia en el contexto académico (Arbeláez y Onrubina, 2014; Sassmannshausen y Volkmann, 2013). Este análisis se caracteriza por su sistematicidad en la recuperación y extracción de datos, Manchado Garabito et al. (2009) al igual que Virgen Ortiz, Cobo Oliveros y Betancourt Guerrero (2014) desarrollan en sus respectivos estudios la serie de decisiones metodológicas que permiten realizar el análisis:

- Manchado Garabito et al. (2009): 1) Definir criterios de inclusión; 2) Identificar las fuentes de información; 3) Establecer las estrategias de búsqueda; 4)
Selección y clasificación de las publicaciones; 5) Definición de las variables de estudio; 6) Extracción de datos.

- $\quad$ Virgen Ortiz et al. (2014): 1) Definición de las categorías de búsqueda; 2) Identificación de bases de datos; 3) Puesta a prueba de las categorías seleccionadas; 4) Ajuste de los criterios de búsqueda; 5) Selección de la base de datos; 6) Recopilación de las categorías de información bibliográfica; 7) Selección de los indicadores bibliométricos a utilizar; 8) Análisis de la información.

Sin embargo, a pesar de la sistematicidad del análisis bibliométrico y la posibilidad de cuantificar la producción científica para su posterior análisis, el contenido de la literatura no es abordado a profundidad, por lo que Arbeláez y Onrubina (2014) proponen una revisión sistemática exploratoria a la par que un análisis de contenido de la literatura identificada, lo que permitiría tener una visión más completa del trabajo que realiza la disciplina. El análisis de contenido tiene como objetivo identificar los componentes de los objetos de comunicación como lo son documentos escritos, permitiendo clasificar la comunicación bajo categorías a fin de describir, medir, identificar o comparar a los textos entre sí (Fernández, 2002).

Dicho lo anterior, la combinación de ambas estrategias permitiría la construcción precisa del cuerpo de contribuciones científicas que la psicología ha desarrollado en torno al emprendimiento. Por tanto, es objetivo del presente estudio analizar el trabajo de la psicología bajo un enfoque sistemático y cuantitativo de la literatura, con el fin de identificar lagunas en la investigación del emprendimiento por parte de la disciplina en las que se pudiera insertar una nueva propuesta conceptual. 


\section{Método}

\section{Análisis Bibliométrico}

Para el análisis bibliométrico, el presente estudio se basó en la metodología de revisiones sistemáticas exploratorias propuesta por Manchado-Garabito et al. (2009), así como en la metodología de Virgen Ortiz et al. (2014). Las decisiones metodológicas se presentan a continuación:

Definición de categorías de búsqueda: Por acuerdo de los autores y con base en el objetivo del presente estudio y sus temáticas de interés, se especificaron las categorías requeridas para la búsqueda de información: palabras clave entrepreneur, entrepreneurship, psychology; periodo de estudio 1980 a la fecha; tipo de fuentes journals bajo peer review; idiomas inglés y español.

Definición de criterios de inclusión y exclusión: Para poder atender el objetivo del presente trabajo, se tomaron en cuenta los siguientes criterios de inclusión:

\section{CRITERIOS DE INCLUSIÓN:}

a) Sobre fuentes de información: Estos criterios buscaron depurar la información con base en fuentes confiables de textos científicos, tales como:

i. Base de datos internacional de textos científicos que incluya literatura de ciencias sociales, en específico de psicología, con el objetivo de examinar la producción científica de la psicología a nivel global en torno al emprendimiento.

ii. Textos científicos arbitrados bajo políticas de revisión por pares, con la finalidad de garantizar la calidad en cuanto a proceso de publicación editorial de los textos científicos recopilados.

b) Sobre tipo de información: Se buscó depurar la información a obtener especificando el tipo de texto científico a analizar, tomando en cuenta:

i. Producción científica contenida en Journals referentes a artículos científicos, con el objetivo de incluir textos científicos dinámicos en cuanto a su producción y contribución.

ii. Textos científicos en idioma inglés y español, con la finalidad de garantizar la inclusión de información de la comunidad científica internacional en cuanto a su producción científica.

c) Sobre el contenido de textos científicos: Estos criterios de inclusión buscaron depurar los textos científicos con base en su contenido orientado efectivamente al estudio del emprendimiento, tales como:

i. Textos científicos que mencionen en su título entrepreneurship, entrepreneur, new Ventures, self-employed, con la finalidad de delimitar a los estudios con base en su temática principal a fin de recopilar únicamente los textos enfocados al emprendimiento desde sus tesauros.

ii. Textos científicos cuyo objetivo esté relacionado con emprendimiento o que mencionen entrepreneurship, entrepreneur, new ventures, selfemployed, con la finalidad de descartar textos científicos cuyo objetivo difiera del estudio del emprendimiento pero que mencionen las palabras clave. 
iii. Inclusión de textos científicos en los que participen una muestra de emprendedores, y de esta manera garantizar la inclusión de artículos interesados en el fenómeno de emprendimiento aun si esto no se declara explícitamente en las demás secciones del texto científico.

\section{CRITERIOS DE EXCLUSIÓN:}

a) Sobre textos científicos: Estos criterios permitieron depurar la muestra con base en aquella información que faltante que obstaculizara el análisis de la literatura, tales como:

i. Información clasificatoria del artículo incompleta en base de datos, con lo cual se excluyeron textos científicos que no permitirían el análisis bibliométrico preciso.

ii. Abstract del artículo no disponible, con lo cual se buscó delimitar el análisis bibliométrico y de contenido a textos científicos con información completa.

iii. Mención de la palabra emprendimiento en la discusión sin conexión directa con el objetivo, excluyendo así textos científicos ajenos a un objetivo directo sobre emprendimiento.

Elección de base de datos: La Biblioteca Digital de la Universidad Nacional Autónoma de México permite el acceso de manera remota a bases de datos nacionales e internacionales referentes a ciencias sociales. El catálogo de bases de datos es amplio, pero la elección se realizó con base en los criterios de inclusión expuestos anteriormente para el apartado de fuentes de información. La base de datos elegida fue Scopus la cual garantiza un proceso de curación bibliográfica sistemática y actualizada de más de 5,000 editoriales internacionales y 16,000 revistas científicas revisadas por pares sobre ciencias médicas, de la salud y ciencias sociales, entre ellas psicología.

Prueba de categorías de búsqueda: Se realizó una prueba de categorías para refinar la búsqueda en la base de datos en marzo del 2018. El histórico de consultas es el siguiente:

1. Búsqueda de palabra clave entrepreneur. Resultado 96,966 textos científicos.

2. Búsqueda de palabra clave entrepreneur en área temática Psychology. Resultado 3, 260 textos científicos.

3. Búsqueda de palabras clave entrepreneur y entrepreneurship añadiendo el operador OR entre los términos. Resultado 186, 839 textos científicos.

4. Búsqueda de palabras clave entrepreneur y entrepreneurship añadiendo el operador OR entre los términos delimitando el área temática Psychology. Resultado 6,146 textos científicos.

5. Búsqueda de palabras clave entrepreneur y entrepreneurship añadiendo el operador AND entre los términos. Resultado 45,098 textos científicos.

6. Búsqueda de palabras clave entrepreneur y entrepreneurship añadiendo el operador AND entre los términos delimitando el área temática Psychology. Resultado 1,351 textos científicos.

La búsqueda seleccionada de palabras clave y operadores fue la prueba número 6 , y la aplicación de todos los demás criterios de inclusión dio una muestra total de 532 artículos científicos para esta revisión sistemática exploratoria, tal como se muestra en la figura 1 . 
Selección de variables/Indicadores bibliométricos: Para el presente estudio se analizaron los siguientes indicadores mencionados por Manchado Garabito et al. (2009): publicaciones por año, publicaciones por país, publicaciones por fuente, publicaciones por institución, autores destacados y su número de citas.

Extracción y análisis de datos: Para realizar el análisis se utilizó el propio motor de búsqueda de Scopus, así como su funcionalidad de análisis incorporada. De igual manera, se utilizaron las consultas de Scopus en texto separado por comas al igual que hojas de cálculo para analizar y graficar la información.

\section{Análisis de Contenido}

Para el análisis de contenido la metodología retomada es la expuesta por Abela (2002): 1) determinar el objeto y tema de análisis; 2) determinar las reglas de codificación; 3) determinar el sistema de categorías; 4) comprobar el sistema de codificación-categorización; 5) analizar los resultados.

Delimitación de unidades de análisis: Para garantizar el análisis de la bibliografía más representativa de la psicología en torno al tema de emprendimiento, se especificaron los siguientes criterios de inclusión:

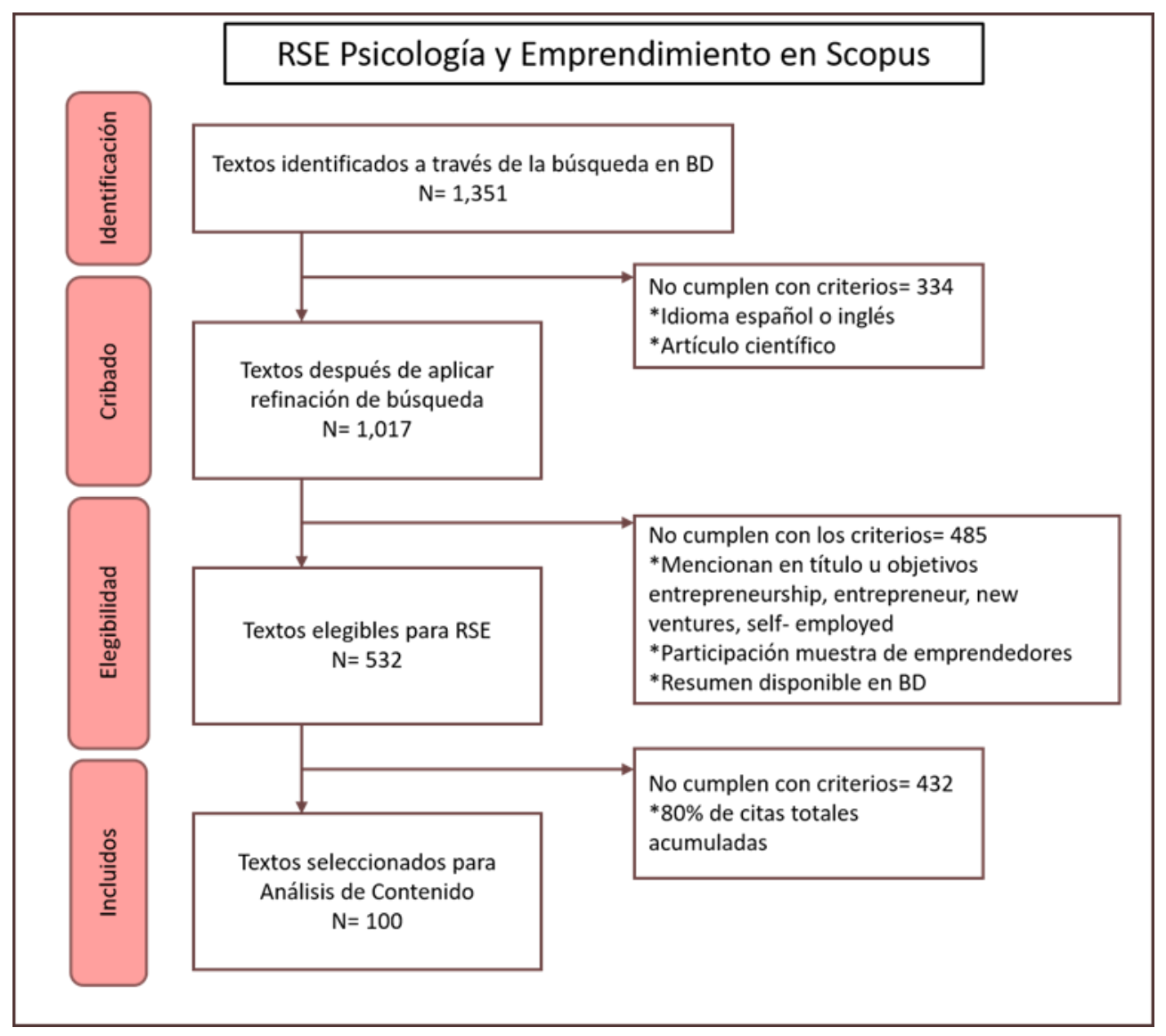

Figura 1. Metodología PRISMA para la identificación, cribado, elegibilidad e inclusión de textos científicos para RSE. 
- Resúmenes de textos científicos resultado de la aplicación de los filtros en la fase de análisis bibliométrico.

- Resúmenes de textos científicos más citados por la comunidad científica.

- Resúmenes con el $80 \%$ acumulado de citas por la comunidad científica.

Muestra de análisis: Posterior a la aplicación de todos los criterios de inclusión, tanto del análisis de bibliométrico como del análisis de contenido, la muestra total de resúmenes de artículos científicos para analizar fueron 100 , los cuales contenían el 80\% acumulado de citas de la comunidad científica (ver Figura 1.).

Sistema de codificación: Para la consolidación de un sistema de codificación, el presente estudio tomó en consideración el propuesto

Tabla 1

Propuesta de categorías para el análisis de la literatura científica.

\begin{tabular}{|c|c|c|}
\hline Nivel de análisis & Categoría & Subcategoría \\
\hline En la persona: & Rasgos & $\begin{array}{l}\text {-Perfil de personalidad } \\
\text {-Perfil sociodemográfico }\end{array}$ \\
\hline $\begin{array}{l}\text { Características permanentes o esta- } \\
\text { dos variables del individuo que } \\
\text { permiten distinguirlo del resto de la } \\
\text { sociedad. }\end{array}$ & Estados & $\begin{array}{l}\text {-Características biológicas } \\
\text {-Salud mental/física } \\
\text {-Habilidades } \\
\text {-Conocimiento/Educación } \\
\text {-Comportamiento orientado al emprendi- } \\
\text { miento } \\
\text {-Orientación/Intención emprendedora }\end{array}$ \\
\hline & Proceso Emprendedor & $\begin{array}{l}\text {-Motivación/Intención } \\
\text {-Orientación emprendedora } \\
\text {-Gestión del proyecto emprendedor }\end{array}$ \\
\hline Contexto social inmediato: & Primario & $\begin{array}{l}\text {-Familia } \\
\text {-Amigos }\end{array}$ \\
\hline $\begin{array}{l}\text { Círculo primario de interacción } \\
\text { para el emprendedor, en el cual el } \\
\text { sujeto puede influenciar y ser in- } \\
\text { fluenciado. }\end{array}$ & Secundario & $\begin{array}{l}\text {-Formales } \\
\text {-Informales }\end{array}$ \\
\hline Contexto político social: & Cultural & $\begin{array}{l}\text {-Grupo étnico } \\
\text {-Valores sociales }\end{array}$ \\
\hline $\begin{array}{l}\text { Contexto más amplio en el que se } \\
\text { encuentra el emprendedor y del }\end{array}$ & Político/Económico & -Contexto local/regional/nacional \\
\hline cual carece de control. & Institucional & $\begin{array}{l}\text {-Instituciones gubernamentales } \\
\text {-Instituciones de incubación } \\
\text {-Instituciones educativas } \\
\text {-Centro laboral }\end{array}$ \\
\hline
\end{tabular}

por Gorgievski y Stephan (2016), en el cual la investigación de la psicología se basa en distintos niveles de análisis, de lo personal o inherente al propio emprendedor a lo macro contextual. De igual manera, se tomaron en cuenta las aportaciones de Rodríguez (2009), Rosero y Molina (2008), así como de Veciana (2007) al constituir un marco de referencia en la investigación de la psicología al emprendimiento. El sistema de categorías resultante se presenta a continuación en la tabla 1 .

Tratamiento de los datos: Para realizar el análisis de contenido se hizo uso del programa de análisis Atlas ti en su versión 7. Para esto se procedió a la extracción de resúmenes filtrados directamente de Scopus, su montaje en el programa de análisis, codificación interna y análisis cuantitativo descriptivo posterior. 


\section{Resultados}

\section{Análisis Bibliométrico}

Al aplicar el filtro entrepreneurship AND entrepreneur SUBJAREA (PSYC), se obtuvieron un total de 1,351 textos científicos. Al aplicar el criterio de idioma (español e inglés) así como el criterio de fuente (artículo científico) el resultado se redujo a 1,017 textos científicos que trataban de emprendimiento desde la disciplina psicológica. Para continuar con el análisis bibliométrico se aplicaron los criterios de inclusión y exclusión de textos científicos especificados en el método, depurando de esta manera la base de datos dando un total de 532 artículos científicos de psicología que efectivamente estudiaban la temática del emprendimiento (ver Figura 1). A continuación, se es- pecifican los resultados por indicador de análisis bibliométrico.

\section{Publicaciones por año}

La muestra depurada de 532 artículos científicos que efectivamente estudian el emprendimiento se distribuye de 1980 a la fecha, presentando en la figura 2 los artículos científicos por año. Con esta muestra de textos científicos es posible observar que es hasta el año de 1990 que la frecuencia de publicación es constante, presentando en 1997 un crecimiento notable que fluctuaría hasta 8 años después en el 2005, año en el que no se detendría el crecimiento $\mathrm{y}$, al contrario, iría en aumento constante hasta la fecha del presente análisis.

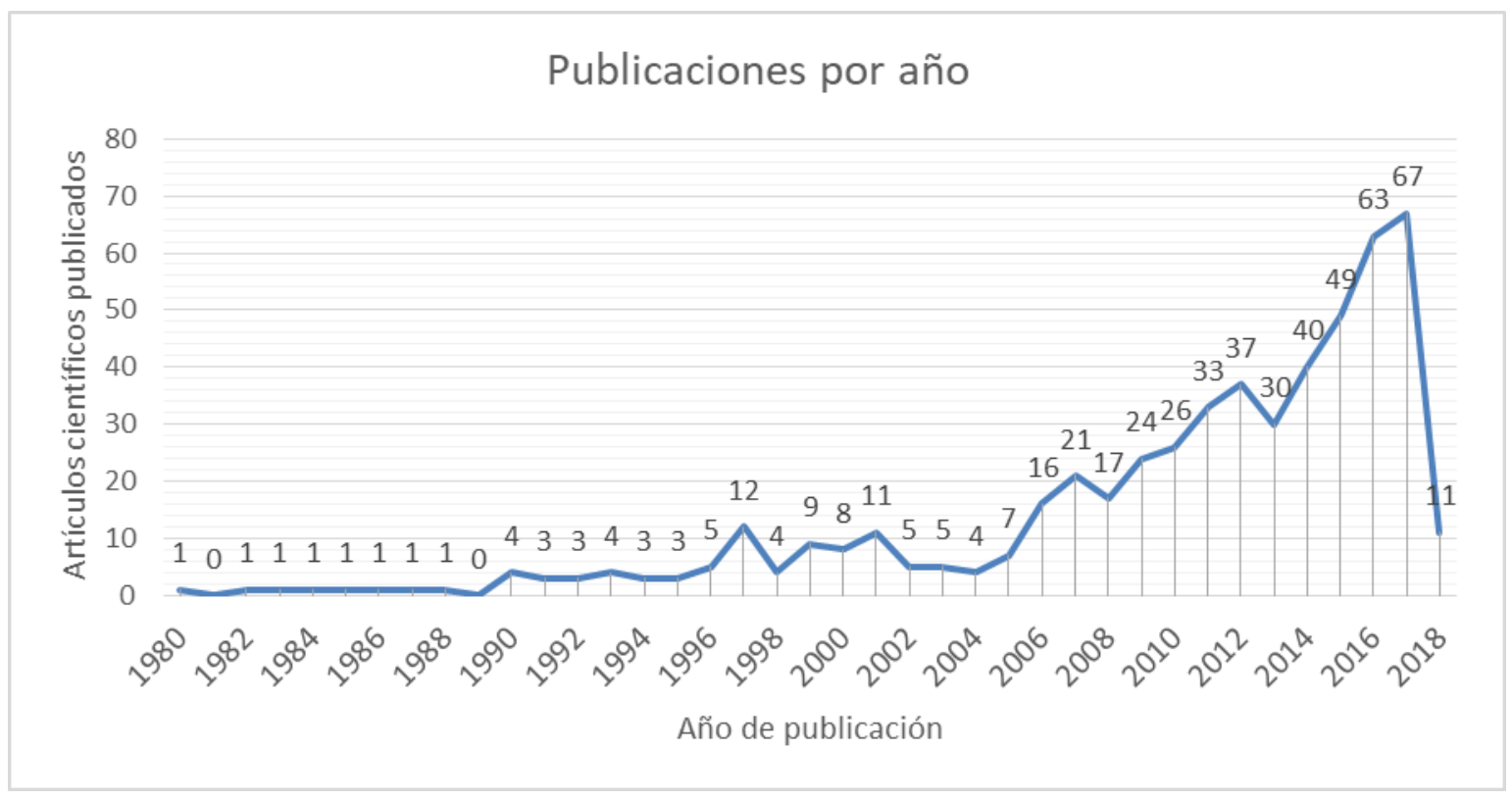

Figura 2. Representación gráfica de la publicación anual de artículos científicos de la psicología que efectivamente estudian el emprendimiento. 


\section{Publicaciones por país}

En el caso de la producción científica por país o territorio, vemos en la figura 3 que el país más productivo es Estados Unidos de América con 177 artículos científicos dedicados al emprendimiento. De igual modo se puede observar como España y Reino Unido presentan una cantidad similar de contribuciones al campo, siendo España el único país hispanohablante con presencia en este ranking.

\section{Publicaciones por Fuente}

Para el caso de publicaciones por fuente, se presenta a continuación la figura 4 que muestra el número de artículos científicos del área de psicología enfocados al emprendimiento. Para este indicador se tomaron en cuenta las fuentes de artículos científicos con más de 10 artículos publicados, filtrando de 39 fuentes sólo a 10 de ellas para su análisis. Es notable en la figura la contribución frecuente de la revista científica internacional dedicada a la administración y ciencias económicas Technological Forecasting And Social Change con 56 artículos científicos sobre emprendimiento del periodo comprendido de 1980 a marzo del presente año.

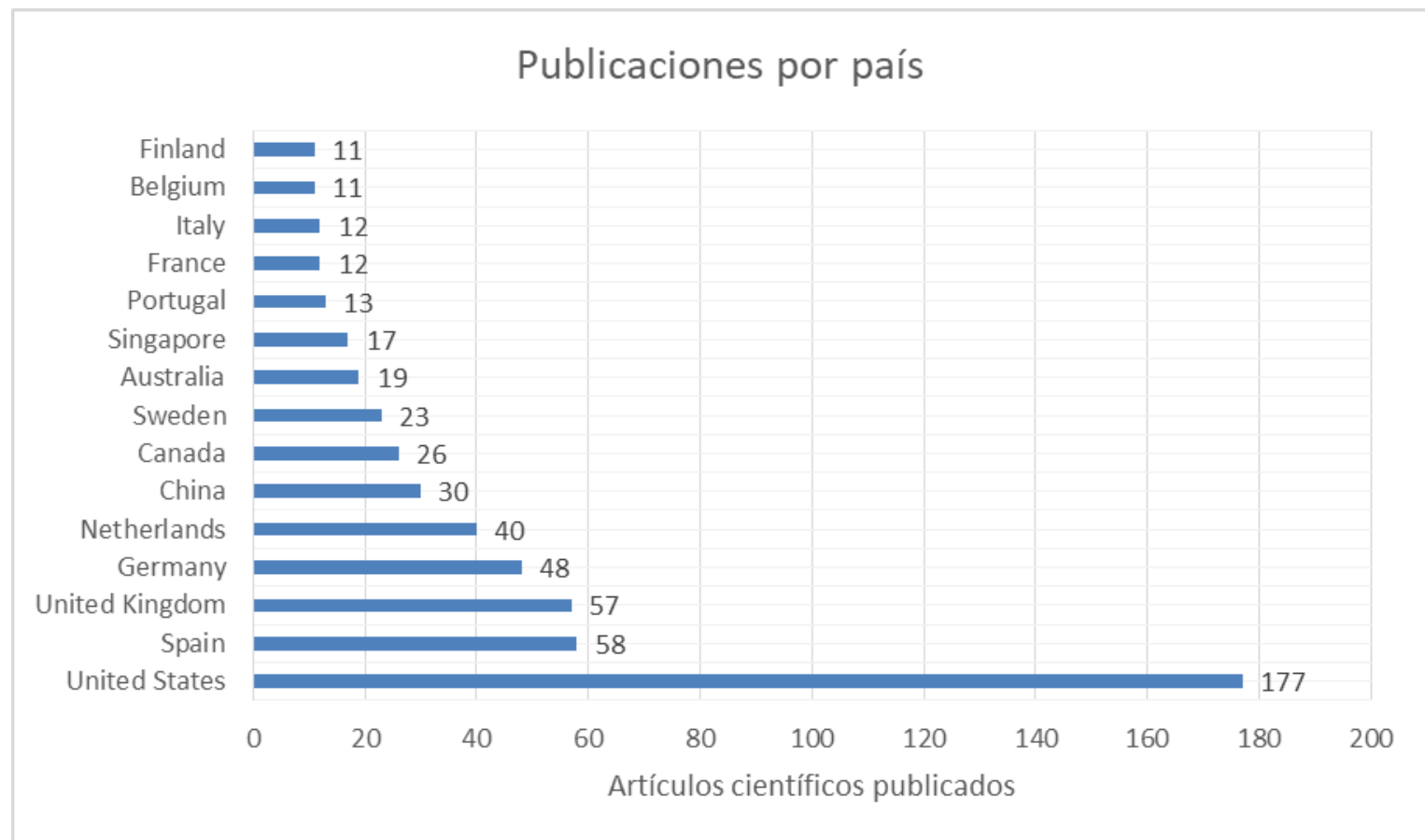

Figura 3. Producción científica por país en el área de la psicología de 1980 a marzo del 2018. 


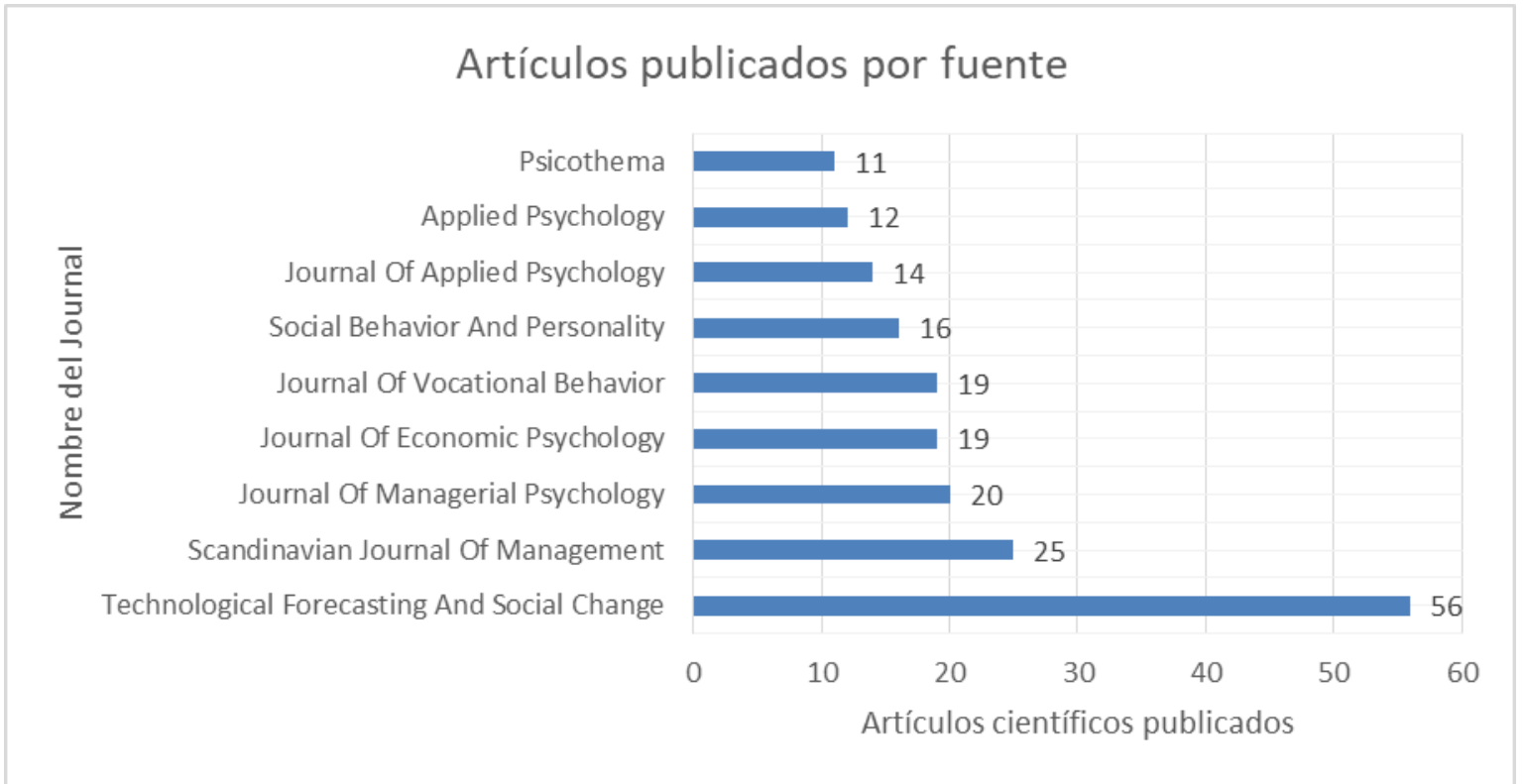

Figura 4. Publicación de artículos científicos por fuente de 1980 a marzo del 2018.

Sin embargo, al analizar con la funcionalidad de la base de datos Scopus el impacto en la comunidad académica de las revistas científicas con mayor número de publicaciones, se obtuvo un gráfico que muestra el CiteScore o puntaje de citas de cada fuente, el cual es un cálculo de las citas que recibe una revista en un año con base en los recursos publicados e indexados a Scopus en los tres años previos al cálculo, permitiendo de esta manera realizar una nueva clasificación basada en este puntaje de impacto ofrecido por Scopus. En este análisis el Journal of Applied Psychology de la American Psychological Association Inc. Representa la fuente de artículos científicos con mayor impacto en la comunidad académica de psicología (figura 5), cabe destacar que el índice de impacto calculado por Scopus contempla todos los textos científicos que publica la fuente, por lo que no es un dato representativo del impacto concreto de los artículos con temática de emprendimiento.
Autores destacados, número de citas y datos de filiación

Para el análisis de contribuciones por autor, se presenta primero la lista de autores destacados por su número de artículos publicados de 1980 a la fecha (figura 6). Para realizar el gráfico se tomaron en cuenta a los primeros 15 autores los cuales acumulaban más producción científica en la materia. Cabe destacar que el número de artículos no necesariamente es en primer orden de autoría, representa únicamente una frecuencia acumulada de aparición en la base de datos como autor de artículo científico de psicología en el estudio del emprendimiento.

Para analizar con mayor detenimiento la producción científica de los autores de este apartado, se presenta en la tabla 2 la lista de los primeros cinco autores, su institución de filiación, el número de artículos publicados, el número de artículos como primer autor, y las líneas de investigación de sus contribuciones: 


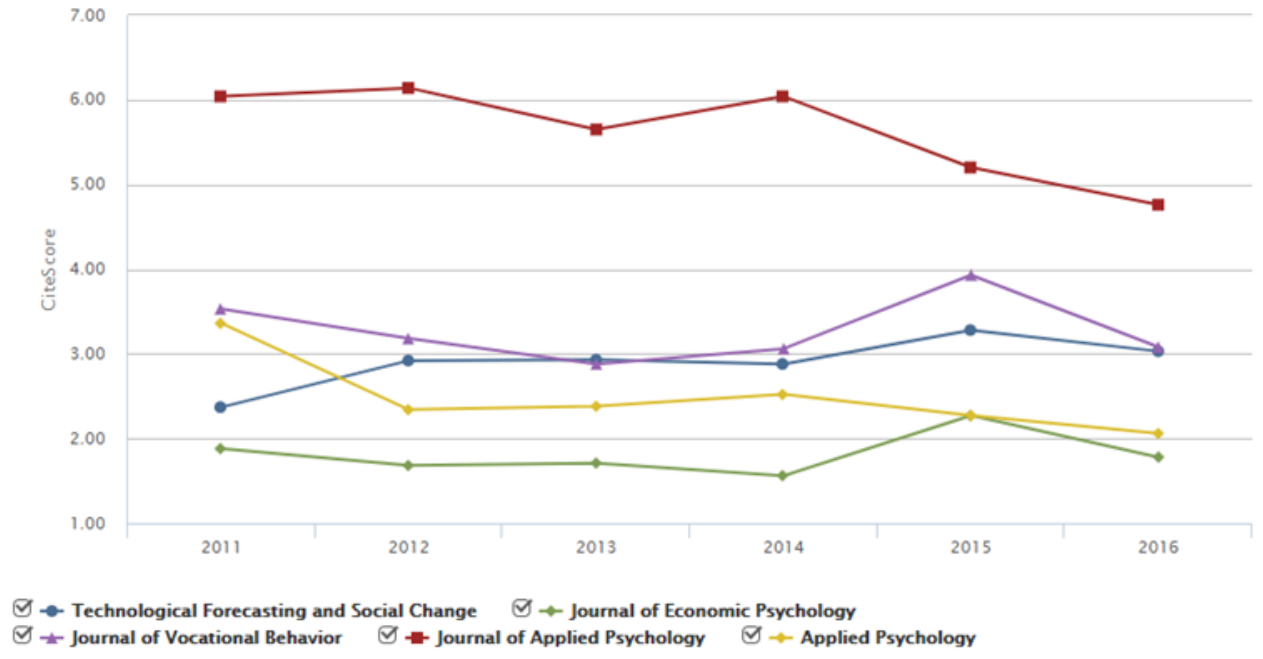

Figura 5. Fuentes con mayor CiteScore calculado por Scopus hasta 2016. La base de datos advierte que no se contemplan las citas anteriores a 1996. Fuente: consulta a base de datos Scopus.

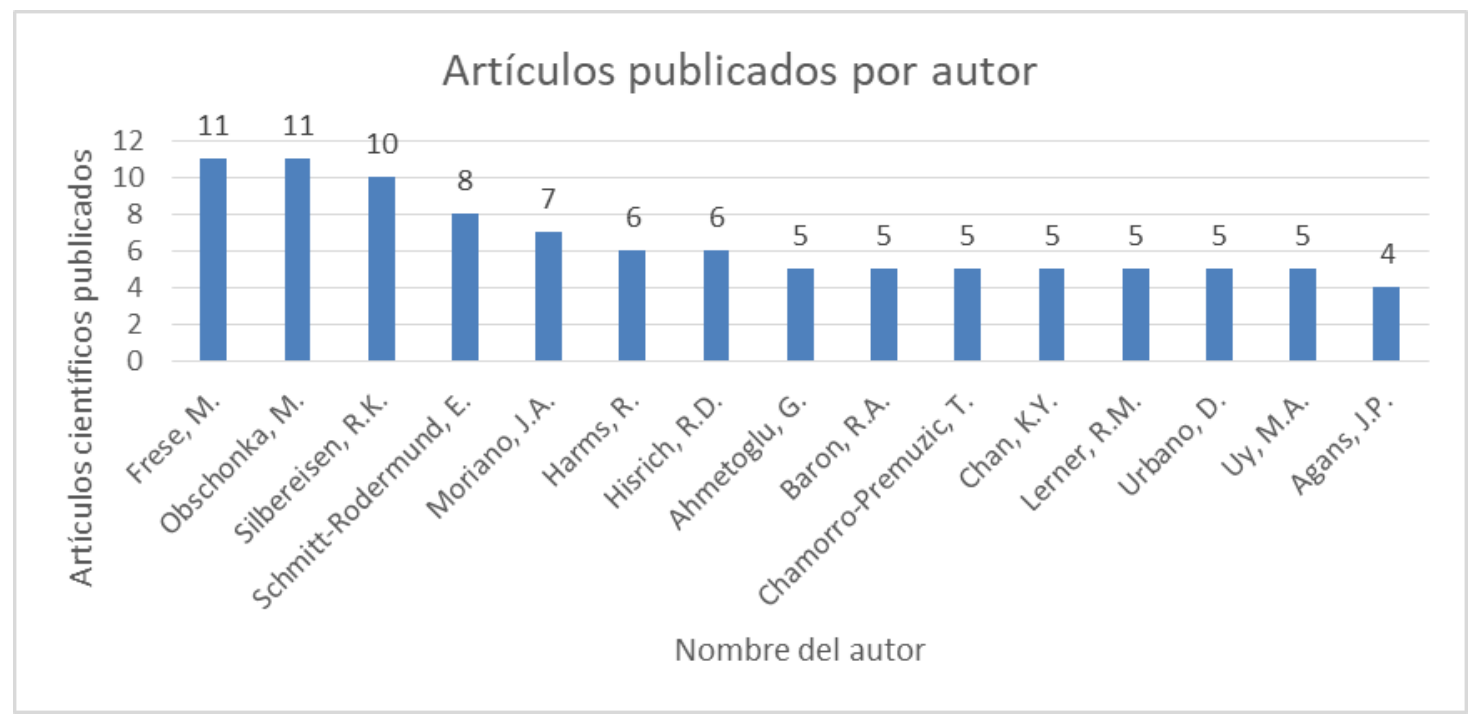

Figura 6. Artículos científicos enfocados al emprendimiento por autor. 
Tabla 2.

Autores destacados por su producción científica extraída de Scopus.

\begin{tabular}{llll}
\hline & Institución de afilia- & Artículos \\
ción & $\begin{array}{l}\text { Artículos } \\
\text { publicados }\end{array}$ & $\begin{array}{l}\text { publicados } \\
\text { como pri- } \\
\text { mer autor }\end{array}$ \\
\hline
\end{tabular}

National University of

City, Singapore

11

4

Iniciativa personal, orientación emprendedora, rasgos de personalidad, acción planeada, desarrollo de carrera emprendedora

Intención emprendedora, emprendimiento nato $\mathrm{y}$ desarrollado, identificación a grupo, determinantes psicológicos y económicos del emprendi-

Friedrich Schiller Uni-

Obschonka, Martin versitat Jena, Jena, 11 Germany

Friedrich Schiller Uni-

Silbereisen, Rainer K. versitat Jena, Jena, 10 Germany

Schmitt-Rodermund, Eva

Friedrich Schiller Universitat Jena, Jena,

8 Germany

Universidad Nacional de educación a DistanMoriano, Juan A. cia, Department of

Social and Organizational Psychology, Madrid, Spain 2 miento, comportamiento emprendedor, competencias del emprendedor, perfil de personalidad, emprendimiento y conductas antisociales delictivas, teoría del comportamiento planeado, emprendimiento en niños y adolescentes.

Intención emprendedora, emprendimiento nato $\mathrm{y}$ desarrollado, desarrollo emprendedor, identificación a grupo, emprendimiento académico, comportamiento emprendedor, emprendimiento en niños y adolescentes, perfil de personalidad del emprendedor, emprendimiento y conducta antisocial delictiva, teoría del comportamiento planeado.

Aspiraciones emprendedoras en adolescentes, paternidad y emprendimiento, competencias tempranas de emprendimiento, rol de personalidad parental, intención emprendedora, emprendimiento nato o desarrollado, desarrollo del emprendimiento, comportamiento emprendedor, perfil de personalidad del emprendedor.

Perfil psicosocial del emprendedor, valores y emprendimiento, psicometría y emprendimiento, autoeficacia emprendedora, conducta intraemprendedora, autoeficacia para liderazgo emprendedor, adicción al trabajo y desempeño del emprendedor, emprendimiento y género, intención emprendedora. 
Como se puede observar, los autores Frese y Obschonka tienen el mismo número de artículos publicados, sin embargo, el número de artículos como primer autor da la ventaja a Obschonka Martin, posicionándolo como el autor más productivo en el tema de emprendimiento desde la psicología. Por otra parte, las líneas de investigación de Obschonka, Silbereisen y Schmitt-Rodermund son similares, esto es debido a que los artículos publicados comparten autoría en distinto orden. Se puede inferir con esta revisión que forman parte de un equipo de investigación que contribuye al estudio del emprendimiento desde la Friedrich Schiller Universitat en Alemania.

Prosiguiendo con el análisis, el autor
Moriano de la Universidad Nacional de Educación a Distancia ocupa el quinto puesto de la lista con 7 artículos publicados, 4 de ellos como autor principal. Entre sus temáticas podemos observar el uso de la psicometría para la medición de propiedades psicológicas referentes al emprendimiento, como lo es la autorregulación y el liderazgo, representando el primer autor en la lista que aborda estas temáticas.

Por otra parte, para conocer un poco más sobre las características de los principales exponentes de la temática por su número de publicaciones, se realiza a continuación una revisión de frecuencias de publicación por institución de procedencia (figura 7).

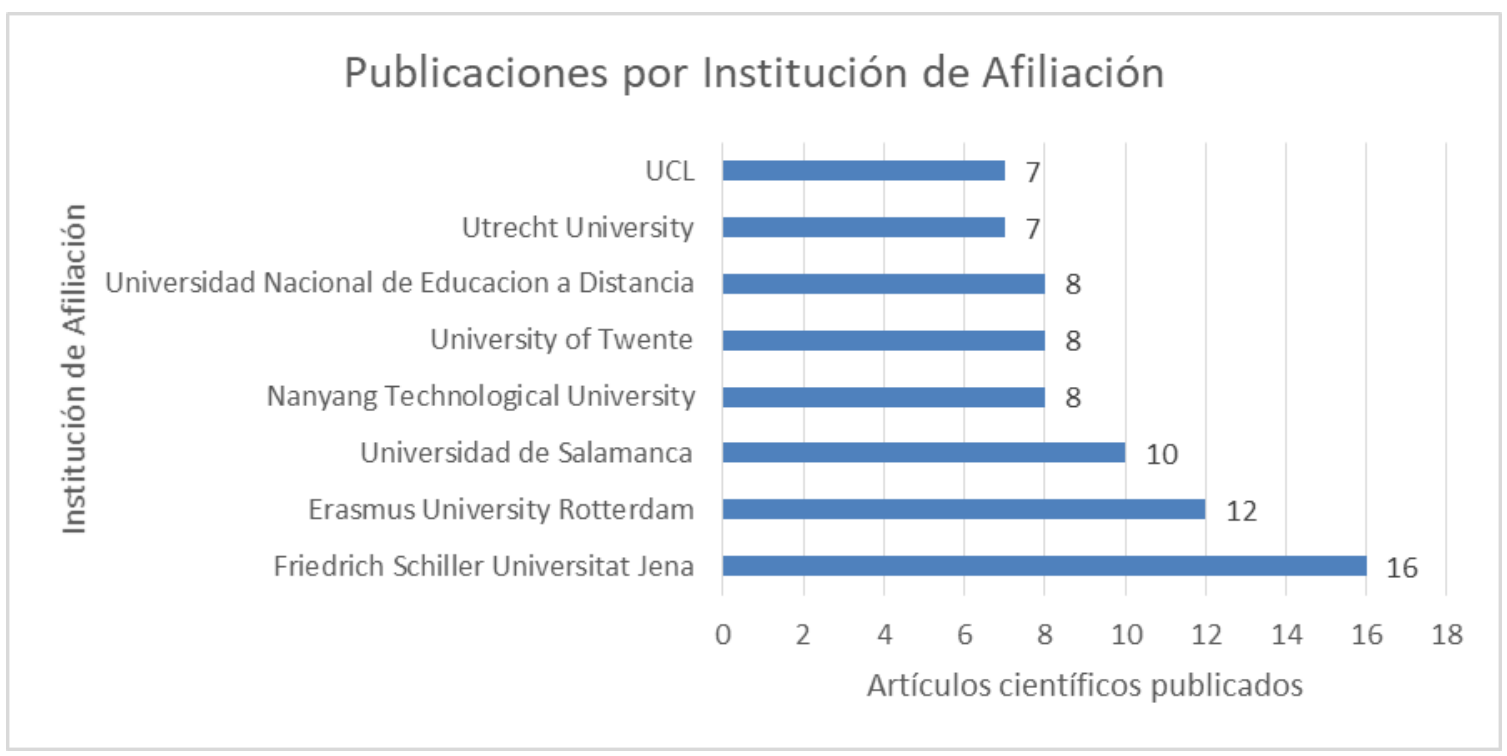

Figura 7. Artículos científicos enfocados al emprendimiento por institución académica de afiliación. 
Reiterando lo encontrado con el indicador anterior, la institución que aporta mayor número de publicaciones científicas sobre emprendimiento es la Friedrich Schiller Universitat en Alemania con un total de 16 publicaciones científicas de 1980 a la fecha, específicamente del 2004 a la actualidad. En el mismo orden de ideas, las instituciones que destacan por su número de contribuciones corresponden a Holanda en segundo lugar, y a España en tercero respectivamente.

Como se ha podido revelar hasta este punto, la relevancia de un autor o institución pública ha sido analizada bajo del número de publicaciones científicas a la fecha. Sin embargo y como se revisó anteriormente, el impacto de una revista, una institución académica o un autor y sus publicaciones científicas, están en función de la aceptación que la comunidad científica realiza de sus aportaciones. Al reconocer la relevancia del aporte científico, éste es citado y diseminado a los académicos interesados en el tema. Por tal motivo, a continuación, se presenta el análisis de frecuencia de citas por artículo y autor de las primeras 15 contribuciones ordenadas por número de citas, lo que permitirá identificar las contribuciones más aceptadas por la comunidad científica (tabla 3 ).

\section{Análisis de contenido}

Análisis de contenido inductivo cualitativo Para analizar con más detalle los textos científicos se realizaron dos tipos de análisis de contenido, uno inductivo a modo exploratorio y otro deductivo a modo confirmatorio. En primer lugar, se realizó el análisis de contenido tipo inductivo a las 15 aportaciones más citadas por la comunidad científica, pues como indica Colle (2011), este tipo de análisis se realiza en conjuntos reducidos de discursos con el objetivo de indagar en los elementos que subyacen a lo manifiesto, lo cual coadyu- vó a identificar de manera interpretativa códigos y categorías con base en la tendencia o frecuencia de ciertas líneas de investigación (Muñóz-Justicia y Sahagún-Padilla, 2017).

El análisis de contenido inductivo/ cualitativo permitió generar un mapeo provisional de las líneas de investigación en la producción científica sobre emprendimiento, tomando en cuenta la aceptación de las contribuciones científicas por la comunidad académica. Cabe destacar que esta aceptación es inferida por la frecuencia de citación de los artículos analizados (tabla 4).

Las temáticas más frecuentemente desarrolladas en esta pequeña lista de artículos relevantes por número de citas van encaminadas a dos niveles de análisis mencionadas por Gorgievski y Stephan (2016) y especificadas en el sistema de categorías para el análisis de contenido, lo cual confirma preliminarmente la clasificación propuesta.

Para profundizar en esto y así poder confirmar la clasificación propuesta sobre la tendencia de investigación, se presentan a continuación los resultados del análisis de contenido deductivo realizado a los abstract de esta muestra de 523 artículos científicos.

\section{Análisis de contenido deductivo}

\section{cuantitativo}

Se ordenó la consulta de textos científicos en la base de datos Scopus por mayor número de citas. El orden permitió identificar los textos académicos más retomados por la comunidad científica, siendo los primeros 100 los que contenían el 80\% acumulado de citas (ver Figura 1).

Para comenzar el análisis se cargaron los resúmenes extraídos de Scopus al programa Atlas ti, realizando en un primer momento un análisis de palabras más frecuentes en todos los resúmenes. 
Tabla 3.

Primeras quince contribuciones científicas al campo del emprendimiento, ordenadas con base en citas recibidas

\begin{tabular}{|c|c|c|c|c|c|}
\hline Lugar & Título de la obra & Autores & $A \tilde{n} \boldsymbol{O}$ & Fuente & Citas \\
\hline 1 & $\begin{array}{c}\text { A Domain-specific Risk- } \\
\text { attitude Scale: Measuring } \\
\text { Risk Perceptions and Risk } \\
\text { Behaviors }\end{array}$ & $\begin{array}{l}\text { Weber, E.U., Blais, A.- } \\
\text { R., Betz, N.E. }\end{array}$ & 2002 & $\begin{array}{l}\text { Journal of Behavioral } \\
\text { Decision Making }\end{array}$ & 851 \\
\hline 2 & $\begin{array}{l}\text { Negative self-efficacy and } \\
\text { goal effects revisited }\end{array}$ & Bandura, A., Locke, E.A. & 2003 & $\begin{array}{l}\text { Journal of Applied } \\
\text { Psychology }\end{array}$ & 786 \\
\hline 3 & $\begin{array}{l}\text { The entrepreneur's business } \\
\text { model: Toward a unified per- } \\
\text { spective }\end{array}$ & $\begin{array}{c}\text { Morris, M., Schindehutte, } \\
\text { M., Allen, J. }\end{array}$ & 2005 & $\begin{array}{c}\text { Journal of Business Re- } \\
\text { search }\end{array}$ & 697 \\
\hline 4 & $\begin{array}{l}\text { The mediating role of self- } \\
\text { efficacy in the development } \\
\text { of entrepreneurial intentions }\end{array}$ & $\begin{array}{l}\text { Zhao, H., Hills, } \\
\text { G.E., Seibert, S.E. }\end{array}$ & 2005 & $\begin{array}{l}\text { Journal of Applied } \\
\text { Psychology }\end{array}$ & 613 \\
\hline 5 & $\begin{array}{l}\text { Personal initiative: An active } \\
\text { performance concept for } \\
\text { work in the } 21^{\text {st }} \text { century }\end{array}$ & Frese, M., Fay, D. & 2001 & $\begin{array}{c}\text { Research in Organizatio- } \\
\text { nal Behavior }\end{array}$ & 464 \\
\hline 6 & $\begin{array}{l}\text { Social skill and institutional } \\
\text { theory }\end{array}$ & Fligstein, N. & 1997 & $\begin{array}{c}\text { American Behavioral } \\
\text { Scientist }\end{array}$ & 457 \\
\hline 7 & $\begin{array}{l}\text { Let's put the person back into } \\
\text { entrepreneurship research: A } \\
\text { meta-analysis on the relation- } \\
\text { ship between business own- } \\
\text { ers' personality traits, busi- } \\
\text { ness creation, and success }\end{array}$ & Rauch, A., Frese, M. & 2007 & $\begin{array}{c}\text { European Journal of } \\
\text { Work and Organizational } \\
\text { Psychology }\end{array}$ & 397 \\
\hline 8 & $\begin{array}{c}\text { The big five personality di- } \\
\text { mensions and entrepreneurial } \\
\text { status: A meta-analytical re- } \\
\text { view }\end{array}$ & Zhao, H., Seibert, S.E. & 2006 & $\begin{array}{l}\text { Journal of Applied } \\
\text { Psychology }\end{array}$ & 371 \\
\hline
\end{tabular}


Tabla 3.

Primeras quince contribuciones cientificas al campo del emprendimiento, ordenadas con base en citas recibidas (Continuación)

\begin{tabular}{|c|c|c|c|c|c|}
\hline 9 & $\begin{array}{c}\text { Work and family variables, } \\
\text { entrepreneurial career suc- } \\
\text { cess, and psychological well- } \\
\text { being }\end{array}$ & $\begin{array}{c}\text { Parasuraman, S., Purohit, } \\
\text { Y.S., Godshalk, } \\
\text { V.M., Beutell, N.J. }\end{array}$ & 1996 & $\begin{array}{c}\text { Journal of Vocational } \\
\text { Behavior }\end{array}$ & 338 \\
\hline 10 & $\begin{array}{l}\text { The concept of personal initi- } \\
\text { ative: Operationalization, reli- } \\
\text { ability and validity in two } \\
\text { German samples }\end{array}$ & $\begin{array}{c}\text { Frese, M., Fay, } \\
\text { D., Hilburger, T., Leng, } \\
\text { K., Tag, A. }\end{array}$ & 1997 & $\begin{array}{c}\text { Journal of Occupational } \\
\text { and Organizational Psy- } \\
\text { chology }\end{array}$ & 327 \\
\hline 11 & $\begin{array}{l}\text { A longitudinal study of the } \\
\text { relation of vision and vision } \\
\text { communication to venture } \\
\text { growth in entrepreneurial } \\
\text { firms }\end{array}$ & $\begin{array}{c}\text { Baum, J.R., Locke, } \\
\text { E.A., Kirkpatrick, S.A. }\end{array}$ & 1998 & $\begin{array}{l}\text { Journal of Applied } \\
\text { Psychology }\end{array}$ & 253 \\
\hline 12 & $\begin{array}{l}\text { Type of employment, work- } \\
\text { family conflict and well- } \\
\text { being: A comparative study }\end{array}$ & $\begin{array}{c}\text { Parasuraman, } \\
\text { S., Simmers, C.A. }\end{array}$ & 2001 & $\begin{array}{c}\text { Journal of Organizational } \\
\text { Behavior }\end{array}$ & 244 \\
\hline 13 & $\begin{array}{c}\text { "I think I can, I think I can": } \\
\text { Overconfidence and entrepre- } \\
\text { neurial behavior }\end{array}$ & $\begin{array}{l}\text { Koellinger, P., Minniti, } \\
\text { M., Schade, C. }\end{array}$ & 2007 & $\begin{array}{c}\text { Journal of Economic } \\
\text { Psychology }\end{array}$ & 242 \\
\hline 14 & $\begin{array}{l}\text { The importance of vertical } \\
\text { and shared leadership within } \\
\text { new venture top management } \\
\text { teams: Implications for the } \\
\text { performance of startups }\end{array}$ & $\begin{array}{l}\text { Ensley, M.D., Hmieleski, } \\
\text { K.M., Pearce, C.L. }\end{array}$ & 2006 & Leadership Quarterly & 230 \\
\hline 15 & $\begin{array}{l}\text { The Relationship of Achieve- } \\
\text { ment Motivation to Entrepre- } \\
\text { neurial Behavior: A Meta- } \\
\text { Analysis }\end{array}$ & $\begin{array}{l}\text { Collins, C.J., Hanges, } \\
\text { P.J., Locke, E.A. }\end{array}$ & 2004 & Human Performance & 191 \\
\hline
\end{tabular}


Tabla 4.

Análisis de contenido inductivo de las 15 aportaciones científicas más citadas.

\begin{tabular}{|c|c|c|c|}
\hline $\begin{array}{l}\text { Nivel de } \\
\text { análisis }\end{array}$ & Categorías & $\begin{array}{l}\text { Frecuencia de } \\
\text { aparición }\end{array}$ & Temáticas \\
\hline \multirow[t]{5}{*}{ Individual } & $\begin{array}{l}\text {-Características indi- } \\
\text { viduales estáticas }\end{array}$ & 2 & Dimensiones y/o rasgos de personalidad, \\
\hline & $\begin{array}{l}\text {-Características indi- } \\
\text { viduales dinámicas }\end{array}$ & & $\begin{array}{l}\text { Características psicológicas desarrolla- } \\
\text { bles: autoeficacia, motivación de logro, }\end{array}$ \\
\hline & & 12 & $\begin{array}{l}\text { actitud al riesgo, habilidades sociales, } \\
\text { liderazgo. }\end{array}$ \\
\hline & & & $\begin{array}{l}\text { Comportamiento emprendedor. } \\
\text { Bienestar ocupacional }\end{array}$ \\
\hline & $\begin{array}{l}\text {-Proceso emprende- } \\
\text { dor }\end{array}$ & 4 & $\begin{array}{l}\text { Iniciativa personal, visión de negocio, } \\
\text { elección de modelo empresarial. }\end{array}$ \\
\hline \multirow[t]{2}{*}{ Social } & -Familia & 2 & Ocupación familiar, trabajo y familia. \\
\hline & -Colegas & 1 & Liderazgo compartido entre colegas. \\
\hline
\end{tabular}

Tabla 5.

Nube de palabras mayormente mencionadas dentro de los textos científicos $(n=100)$.

\begin{tabular}{|c|c|c|c|c|c|}
\hline Orden & Palabra & Frecuencia & Orden & Palabra & Frecuencia \\
\hline 1 & Business & 107 & 6 & Role & 59 \\
\hline 2 & Study & 72 & 7 & Social & 59 \\
\hline 3 & New & 66 & 8 & Results & 57 \\
\hline 4 & Performance & 61 & 9 & Personality & 57 \\
\hline 5 & Research & 61 & 10 & Venture & 52 \\
\hline
\end{tabular}


De la nube de palabras se descartaron los términos Entrepreneurial, Entrepreneurship y Entrepreneurs, por ser las palabras clave de búsqueda en la base de datos Scopus. Los resultados se expresan en la tabla 5.

Con el conteo de palabras podemos examinar de manera general el contenido de los textos analizados con Atlas ti, lo cual nos habla de una clara orientación de la investigación psicológica más citada hacia el estudio del emprendimiento con una visión empresarial, utilizando términos como negocio, desempeño, rol, social, personalidad y riesgo.

Para proceder a un análisis más detallado del contenido se empleó el análisis de contenido deductivo/cualitativo, el cual se caracteriza por estar orientado al resumen, definición y verificación de categorías de conjuntos amplios de discursos, en este caso resúmenes de artículos científicos (Colle, 2011). Para esto, se identificaron dentro de los abstracts las categorías establecidas en el sistema de categorías desarrollado con base en las aportaciones de Rodríguez (2009), Rosero y Molina (2008), y Veciana (2007), para de esta manera determinar la congruencia que guardó el sistema de categorías propuesto con la literatura más citada en el tema.

De igual modo, para efectos descriptivos se añadieron dos categorías clasificatorias en referencia al tipo de texto que se presenta: estudios teóricos/conceptuales y estudios empíricos. Es así que, al identificar el tipo de estudio (teórico/conceptual vs. empírico; revisión sistemática de la literatura vs. revisión narrativas para el caso de estudios teóricos/ conceptuales, y experimental vs. no experimental para el caso de estudios empíricos), así como su línea de investigación principal, se obtuvieron los siguientes datos:

- $\quad$ El 30\% de la muestra analizada de tex- tos académicos son de tipo teórico/ conceptual. E1 20\% de los estudios teórico/conceptuales se formularon con base en una revisión sistemática. El $80 \%$ de estos estudios constituyen revisiones narrativas de la literatura.

- $\quad$ Para las revisiones sistemáticas, el 50\% de éstas se enfocaron en desarrollar el perfil de personalidad del emprendedor. Mientras que el 30\% los comportamientos característicos del emprendedor y el $20 \%$ restante a la relación que guarda el capital social y el emprendimiento.

- Para el caso de las revisiones narrativas, el $71 \%$ se enfocó en desarrollar posturas teórico-conceptuales en un nivel de análisis del sujeto, es decir a los rasgos o estados del emprendedor. El 17\% se dirigió a conceptualizar o analizar el contexto cultural, político y social del emprendedor, y el $12 \%$ a las relaciones interpersonales del emprendedor.

Por otra parte, el $70 \%$ de textos que componen la muestra fueron de tipo empírico, y de estos escritos empíricos el 94\% fueron estudios no experimentales, mientras que el $6 \%$ fueron de corte experimental los cuales desarrollaban temáticas relacionadas con la ejecución del emprendedor y los valores o ideología social sobre el emprendimiento.

A través del análisis de contenido fue posible comprobar la congruencia del sistema categorial formulado para precisar la dirección de la producción científica en torno al emprendimiento. La variedad de temáticas que se presentaron en los estudios es amplia, y a grandes rasgos respetó la lógica de dirección por niveles de análisis (personal, social inmediato y político social externo).

Sin embargo, hubo categorías que preci- 
saron una adecuación para clasificar el trabajo científico que se ha realizado, tal es el ejemplo de la categoría "Estados", ya que al realizar el análisis de contenido la lógica categorial se refinó pasando de cuatro subcategorías a sólo dos: ejecución y salud mental/física del emprendedor. De igual manera en la categoría "Institucional", se depuró la lista de subcategorías quedando sólo dos: centro laboral e instituciones educativas.

Por tales motivos y con fines expositivos, en la tabla 6 se presentan las categorías con las adecuaciones mencionadas, así como la frecuencia con la que fueron retomadas por el trabajo de investigación tanto empírico como teórico/conceptual, añadiendo de igual manera las temáticas más citadas en cada subcategoría y el autor o autores que exponen la línea de investigación.

Como se puede observar, la subcategoría más desarrollada por la investigación en psicología de 1980 a marzo del 2018 es la referente a la ejecución del emprendedor. En esta subcategoría se responden cuestiones como ¿Qué hace un emprendedor para caracterizarse como tal? Mientras que la segunda subcategoría más frecuentemente desarrollada es la referente a la delimitación de un perfil de personalidad, en la cual se pretende responder a la pregunta ¿Qué rasgos posee un emprendedor para caracterizarse como tal?, ambas contenidas en el nivel de análisis del emprendimiento desde la persona, pero desde distinto enfoque teórico como lo es el de rasgos y estados psicológicos.

Otra de las subcategorías mayormente retomadas para la investigación en torno al emprendimiento es el de características biológicas contenida en la categoría de rasgos al tratarse de una condición fija o predeterminada en el individuo. En esta subcategoría se encuentran temas referentes al sexo o condiciones biológicas que de alguna manera se relacionan con el emprendimiento, como características genéticas $\mathrm{u}$ hormonales. Por otra parte, en la categoría del proceso emprendedor la subcategoría más retomada en las investigaciones analizadas es la referente a la de gestión del proyecto emprendedor, en la cual se retoman temáticas relacionadas con decisiones en torno al modelo de negocio, estrategia de negocio o actividades que permitan la permanencia y desarrollo de la acción emprendedora.

Para el caso del nivel de análisis de contexto social inmediato, la subcategoría más retomada en los textos analizados es la referente a la familia con temáticas en torno a la influencia parental y crianza para la elección del emprendimiento u orientación emprendedora. En este mismo nivel de análisis, se habla de las relaciones sociales formales o informales, que permiten al emprendedor contar con una red social de apoyo en el proceso de emprendimiento empresarial. Por último, el nivel de análisis macro contextual o contexto político y social, las subcategorías más retomadas para la investigación en torno a emprendimiento es la influencia que ejerce el medio social en los distintos niveles del emprendimiento, desde su surgimiento como orientación emprendedora o intención emprendedora, hasta la consolidación del proyecto empresarial. 
Tabla 6.

Resultado del análisis de contenido deductivo con frecuencia de ocurrencia por subcategoría $(n=100)$, así como temáticas más citadas en cada subcategoría.

\begin{tabular}{|c|c|c|c|c|}
\hline $\begin{array}{l}\text { Nivel de } \\
\text { análisis }\end{array}$ & Categoría & Subcategoría & Frecuencia & Temáticas más citadas \\
\hline \multirow[t]{9}{*}{ En la persona: } & Rasgos & Perfil de Personalidad & 23 & $\begin{array}{l}\text { Perfil de personalidad de emprende- } \\
\text { dores que inician un negocio, que } \\
\text { tienen éxito en su negocio, y no em- } \\
\text { prendedores (Frese, 2007). }\end{array}$ \\
\hline & & Perfil sociodemográfico & 2 & $\begin{array}{c}\text { Factores sociodemográficos relacio- } \\
\text { nados con la preferencia al autoem- } \\
\text { pleo (Verheul, Thurik, Grilo y Van } \\
\text { der Zwan, 2012). }\end{array}$ \\
\hline & & Características biológicas & 8 & $\begin{array}{c}\text { Causas del emprendimiento acelerado } \\
\text { de mujeres (Weiler y Bernasek, } \\
\text { 2001). }\end{array}$ \\
\hline & & Historia personal & 5 & $\begin{array}{l}\text { Factores disposicionales del empren- } \\
\text { dimiento e intraemprendimiento rela- } \\
\text { cionados con su historia de vida, edu- } \\
\text { cación y trabajo (Hisrich, 1990). }\end{array}$ \\
\hline & Estados & Salud mental/física & 5 & $\begin{array}{l}\text { Influencia del trabajo y la familia en } \\
\text { el bienestar psicológico de emprende- } \\
\text { dores (Parasuraman, Purohit, } \\
\text { Godshalk y Beutell, 1996). }\end{array}$ \\
\hline & & Ejecución & 46 & $\begin{array}{l}\text { Creencias de autoeficacia en la obten- } \\
\text { ción de objetivos bajo la autorregula- } \\
\text { ción (Bandura y Locke, 2003). }\end{array}$ \\
\hline & $\begin{array}{l}\text { Proceso em- } \\
\text { prendedor }\end{array}$ & Motivación/Intención & 8 & $\begin{array}{l}\text { Motivos que estimulan el ser propie- } \\
\text { tarios de un negocio (Cromie, 1987). }\end{array}$ \\
\hline & & Orientación emprendedora & 5 & $\begin{array}{l}\text { Relación entre éxito empresarial y } \\
\text { orientación emprendedora (Krauss, } \\
\text { Frese, Friedrich y Unger, 2005). }\end{array}$ \\
\hline & & $\begin{array}{l}\text { Gestión del proyecto } \\
\text { emprendedor }\end{array}$ & 13 & $\begin{array}{l}\text { Elección del modelo de negocio y la } \\
\text { práctica emprendedora (Morris, } \\
\text { Schindehutte y Allen, 2003) }\end{array}$ \\
\hline
\end{tabular}


Tabla 6.

Resultado del análisis de contenido deductivo con frecuencia de ocurrencia por subcategoría $(n=100)$, así como temáticas más citadas en cada subcategoría. (Continuación)

\begin{tabular}{|c|c|c|c|c|}
\hline \multirow[t]{3}{*}{$\begin{array}{l}\text { Contexto } \\
\text { social inme- } \\
\quad \text { diato: }\end{array}$} & Primario & Familia & 6 & $\begin{array}{l}\text { Relación entre crianza, competencia em- } \\
\text { prendedora e intereses emprendedores } \\
\text { (Schmitt-Rodermund, 2003). }\end{array}$ \\
\hline & Secundario & Formales & 5 & $\begin{array}{l}\text { Emprendedores institucionales y su influen- } \\
\text { cia en el resto de la organización a través de } \\
\text { las habilidades sociales (Fligstein, 1997) }\end{array}$ \\
\hline & & Informales & 5 & $\begin{array}{l}\text { Desarrollo y mantenimiento de una red so- } \\
\text { cial para el éxito emprendedor } \\
\text { (Johannisson, 1988). }\end{array}$ \\
\hline \multirow[t]{5}{*}{$\begin{array}{l}\text { Contexto } \\
\text { político so- } \\
\text { cial: }\end{array}$} & Cultural & Grupo étnico & 1 & $\begin{array}{c}\text { Competencia étnica e intra-étnica por el } \\
\text { dominio de un nicho de negocio (Lee, } \\
\text { 1999). }\end{array}$ \\
\hline & & $\begin{array}{c}\text { Valores/ } \\
\text { Ideología sociales }\end{array}$ & 8 & $\begin{array}{l}\text { Valores y creencias como determinante } \\
\text { cultural para el nivel de emprendimiento de } \\
\text { una sociedad (Davidsson y Wiklund, 1997). }\end{array}$ \\
\hline & $\begin{array}{l}\text { Político/ } \\
\text { Económico }\end{array}$ & $\begin{array}{l}\text { Contexto local/ } \\
\text { regional/nacional }\end{array}$ & 5 & $\begin{array}{l}\text { Perspectiva socioecológica del emprendi- } \\
\text { miento (Obschonka, Schmitt-Rodermund, } \\
\text { Silbereisen, Gosling y Potter, 2013). }\end{array}$ \\
\hline & Institucional & $\begin{array}{l}\text { Instituciones educati- } \\
\text { vas }\end{array}$ & 1 & $\begin{array}{l}\text { Relación entre educación en emprendimien- } \\
\text { to y actividad emprendedora (Raposo y } \\
\text { Paço, 2011). }\end{array}$ \\
\hline & & Centro laboral & 1 & $\begin{array}{l}\text { Influencia institucional en los administrado- } \\
\text { res para ser emprendedores institucionales } \\
\text { (Rothenberg, 2007). }\end{array}$ \\
\hline
\end{tabular}




\section{Discusión y conclusiones}

La revisión de la literatura que se presenta se realiza con intenciones exploratorias y descriptivas, centradas en la identificación sistemática de las contribuciones científicas al campo del emprendimiento por parte de la psicología; se optó por este método en lugar del narrativo a fin de evitar resultados imprecisos, ya que las revisiones narrativas permiten que sea el criterio del investigador el que guíe la formulación teórica y de evidencia empírica sobre la materia, y por el contrario, el análisis sistemático exploratorio se centra en el trabajo que la disciplina ha realizado efectivamente hasta el momento.

Es así que, la revisión sistemática exploratoria es una estrategia que promete mayor precisión en la identificación de la producción científica debido a la identificación de las publicaciones científicas con base en indicadores bibliométricos, con este ejercicio se atiende a lo efectivamente presente y desarrollado hasta el momento en el campo de estudio, y no a la preferencia o disposición del revisor por determinada corriente o modelo teórico.

A través del análisis bibliométrico se detectó la tendencia productiva de las comunicaciones científicas sobre emprendimiento, por lo menos en la base de datos Scopus y con los criterios de inclusión y exclusión empleados para este estudio. Así se detecta que desde la década de 1990 aumenta el interés de la comunidad científica por la temática yendo en una tendencia positiva desde entonces.

De igual modo, el análisis permitió identificar los países con mayor contribución al campo, siendo Estados Unidos de América el país más productivo en la temática, seguido de España, Reino Unido, Alemania y Países Bajos, entre otros países europeos y asiáticos. En este listado España es el único país de habla hispana con producción científica notable en esta base de datos, casi igualable a la producción de Reino Unido.

Por otra parte, se identificaron las revistas científicas con mayor número de publicaciones sobre emprendimiento, de igual modo, las revistas científicas con mayor impacto basado en el indicador CiteScore ofrecido por Scopus. En este breve análisis podemos identificar que la revista científica Technological Forecasting and Social Change publicada por Elsevier es la más productiva en el campo del emprendimiento, pero la que más bajo CiteScore obtuvo por Scopus. Por el contrario, el Journal of Applied Psychology editada por la American Psychological Association tiene una producción moderada de 1980 a marzo del 2018, sin embargo, presenta el CiteScore más elevado de la lista, lo cual podría indicar que es una de las fuentes más consultadas y retomadas por la comunidad académica en los últimos 10 años, seguida por el Journal of Vocational Behavior editada por Elsevier.

Una vez analizadas las revistas científicas más productivas y consultadas por la comunidad académica, se procedió a identificar a los autores más productivos y sus instituciones de procedencia. En este sentido, la Friedrich Schiller Universitat en Alemania presenta un grupo de trabajo muy productivo representado por Obschonka, Silbereisen y Schmitt-Rodermund, quienes tienen líneas de investigación que giran en torno a la intención emprendedora, emprendimiento nato y desarrollado, determinantes psicológicos para el emprendimiento, comportamiento emprendedor, entre otras, lo cual implica una productividad amplia en términos numéricos y temáticos.

Así mismo, fue posible detectar la notable contribución de Frese, Michael de la $\mathrm{Na}$ tional University of Singapore quien ha publi- 
cado en coautoría y autoría principal en numerosas ocasiones con líneas de investigación como iniciativa personal, orientación emprendedora, rasgos de personalidad, entre otras. De igual modo, se encuentra en la lista el español Moriano, Juan de la Universidad Nacional de ducación a Distancia, Department of Social and Organizational Psychology quien ha contribuido con temáticas como Perfil psicosocial y valores del emprendedor, medición psicométrica del emprendimiento, autoeficacia, entre otras.

Las líneas de investigación, sus autores e instituciones de procedencia permiten analizar las necesidades particulares del entorno de los investigadores, y las respuestas que éstos proporcionan a dichas necesidades. Cabe destacar que lo autores más citados se retomaron de una lista más amplia de investigadores, pero al ser autores de los trabajos más retomados por la comunidad científica habla de igual modo sobre la preferencia temática que gira en torno al emprendimiento, su comprensión y análisis.

Para concluir el análisis bibliométrico, se ofreció un listado de las contribuciones científicas más citadas por la comunidad académica, las cuales tienen una antigüedad de 20 años, pero gozan de reconocimiento por los investigadores en el campo. En este listado encontramos autores como Weber, Bandura, Morris, Zhao, y Frese, éste último revisado en el indicador anterior sobre productividad académica.

De la lista de obras más citadas podemos destacar las temáticas desarrolladas por los investigadores, como lo es la toma de riesgos, autoeficacia positiva y negativa, iniciativa personal y modelo de negocios, lo cual nos habla tanto de las tendencias comportamentales de emprendedores, como de rasgos de personalidad más estudiados en la temática. Esto lo observan autores como Rosero y Molina (2008) así como Veciana (2007), quienes en su identificación temática contemplan la esfera personal del emprendedor y las vertientes de investigación en rasgos y perfiles del emprendedor, al igual que comportamiento orientado al emprendimiento.

Por otra parte, la estrategia de parear la revisión sistemática exploratoria con un análisis de contenido inductivo como deductivo, permitió ubicar los componentes principales de las comunicaciones escritas como lo son los textos científicos. En este caso, la utilización de ambas estrategias de análisis permitió distinguir un ejercicio de la psicología con intereses bien marcados. Sobre esto, se puede constatar que la psicología se ha evocado más a las esferas personal e interpersonal del emprendedor, lo cual corresponde naturalmente a su objetivo disciplinar, encontrando en menor medida la participación de la psicología en temas macro-contextuales como el institucional, político económico y cultural.

Así mismo, el análisis de contenido permitió identificar las necesidades de investigación del campo del emprendimiento, ya que al retomar el tipo de estudio llevado a cabo por los trabajos de esta muestra, se puede detectar una contribución desbalanceada de artículos empíricos no experimentales y experimentales, lo cual implica una posible validez externa alta pero validez interna baja con varias fuentes de sesgos o errores sistemáticos al no controlar las fuentes de invalidez en condiciones experimentales (Kerlinger, 1988).

De igual modo, el porcentaje bajo de estudios experimentales es comprensible al desprenderse la temática de emprendimiento como un fenómeno social, no originario de condiciones de laboratorio. Esta situación representa un área de oportunidad para los investigadores en cualquier temática, ya que al 
tratar de generalizar las conclusiones del proceso de investigación se debe de contar con elementos que permitan responder y replicar las hipótesis planteadas en torno al fenómeno de interés tanto en condiciones naturales como controladas (Barlow y Hersen, 1988).

Sin embargo, el objetivo del presente estudio dista de valorar como positiva o negativa, suficiente o insuficiente la contribución que se ha hecho hasta el momento al campo del emprendimiento. Al contrario, se centra en identificar y reconocer los avances que investigadores, instituciones y organizaciones académicas han realizado en el estudio del fenómeno, para de esta manera insertar una perspectiva teórica que contribuya de igual manera al campo de estudio.

Además, como ejercicio reflexivo del propio proceder es necesario puntualizar que el presente estudio posee por si mismo limitaciones, como lo es centrar el análisis bibliométrico en una sola fuente de información como lo fue Scopus o el haber centrado todo el análisis en un solo tipo de texto académico como artículos científicos, aun así, se buscó sistematizar la definición de criterios, el proceso y el análisis de los resultados para detectar con relativa precisión un área de oportunidad para el estudio del emprendimiento como fenómeno psicológico.

En este sentido, con este ejercicio se buscó detectar el campo de inserción en el que se centra un proyecto más amplio, como lo es la constitución del comportamiento emprendedor en el mapa de estados psicológicos que promueven la acción emprendedora en cualquier ámbito, incluido el empresarial. El comportamiento emprendedor podría definirse como la tendencia comportamental derivada de una serie de competencias encaminadas de manera autodirigida a la modificación de la situación presente de un individuo para el alcance de un criterio de logro en un contexto determinado, el cual puede ser plenamente identificado por el sujeto, como lo es un objetivo o meta personal. Cabe destacar que la "situación presente" no se limita a un contexto empresarial como ha sido la tradición en el estudio del emprendimiento como pudo constatarse en este análisis, sino que atiende a todos los posibles contextos en los que se pueda presentar un criterio de logro por el individuo en un contexto particular. De igual manera, se presenta el criterio de logro como propio del sujeto, pudiendo ser compartido e identificado por el resto de la sociedad, como por ejemplo la constitución de una empresa.

La definición de comportamiento emprendedor se podría tomar como ajena a lo que el propio fenómeno de emprendimiento ha supuesto desde el inicio de su estudio, que es la persecución de un proyecto empresarial con fines lucrativos por parte de un emprendedor; la aparente no correspondencia es comprensible si se analizan las palabras de Freixa i Baqué (2003) en torno a un error que se comete con frecuencia dentro de la psicología, el cual consiste en confundir el fenómeno completo con una conceptualización del propio fenómeno, a extremos en los que ya no se acepta otra explicación que no sea la conceptualización inicial del fenómeno, en este caso, la acuñada desde la economía desde el siglo XVIII (Najaragan, 2011).

De igual manera, se destaca el campo de inserción de esta propuesta conceptual, el cual es enteramente psicológico y no descalifica lo aportado por las ciencias políticas, económicas o administrativas en torno a la gestión de un proyecto empresarial, sólo se asume que la disciplina psicológica puede estudiar el fenómeno desde su propio ámbito de competencia que es el estudio de la conducta humana, para posteriormente ser tratado 
y entendido por otras disciplinas con todos los factores político, económicos y sociales que le corresponden.

Sin embargo, la inserción de esta explicación comportamental y funcional desde la psicología al fenómeno del emprendimiento requiere una serie de comprobaciones que den cuenta de la robustez de su constitución y aplicación, ya que la definición presentada no representa en lo más mínimo una versión final, y debe de ajustarse con una serie de nuevos análisis. Por tanto, como seguimiento a lo realizado en esta identificación inicial de tendencias temáticas, se propone como líneas de acción futuras el desarrollo de un análisis sistemático de la literatura en búsqueda de las características asociadas al fenómeno (Glasziou, Irwig, Bain y Colditz, 2001), y de esta manera determinar la serie de competencias, habilidades, aptitudes o destrezas que componen el comportamiento de un emprendedor, para posteriormente poner a prueba empírica el constructo y sus implicaciones.

En síntesis, se podría decir que el aporte de la psicología al emprendimiento ha sido en un primer lugar, delimitar la ejecución del emprendedor en términos de un criterio de logro que, en este caso, se relaciona con la creación de un proyecto empresarial. En segundo lugar, y con base en lo señalado por Carlsson et al. (2013), se consolida la reflexión de que la psicología ha enfocado sus esfuerzos a determinar los rasgos o características de personalidad que permitan distinguir a un emprendedor de otros que no lo son; pero retomando lo mencionado por Gartner (1988) como crítica a esta práctica, "lo que diferencia a un emprendedor de un no emprendedor, es que el emprendedor crea organizaciones, mientras que el no emprendedor no" (pp. 47) [traducido por la autora]; a esta reflexión, sólo habría que agregar: lo que diferencia a un emprendedor de un no emprendedor, es que el emprendedor consigue su criterio de logro, mientras que el no emprendedor no. Para comprobar esto y determinar las competencias de las cuales se sirve el emprendedor para conseguir su objetivo, se seguirá trabajando.

\section{Referencias:}

Abela, J. A. (2002). Las técnicas de análisis de contenido: una revisión actualizada. Fundación Centro de Estudios Andaluces 1-34. Recuperado de: http://public.centrodeestudiosandaluces.es/ pdfs/S200103.pdf

Arbeláez, M., \& Onrubina, J. (2014). Análisis bibliométrico y de contenido. Dos metodologías complementarias para el análisis de la revista colombiana Educación y Cultura. Revista de Investigaciones. UCM, 14(23), 14-31. doi:10.22383/ri.v14i1.5

Barlow, D. H. \& Hersen, M. (1988). Diseños experimentales de caso único. Estrategias para el estudio del cambio conductual. España: Martínez Roca.

Beltrán, G. (2005). Revisiones sistemáticas de la literatura. Revista Colombiana de Gastroenterología, 20(1), 60-69. Recuperado de: http:// www.scielo.org.co/pdf/rcg/v20n1/ v20n1a09.pdf

Carlsson, B., Braunerhjelm, P., McKelvey, M., Olofsson, C., Persson, L., \& Ylinenpää, H. (2013). The evolving domain of entrepreneurship research. Small Business Economics, 41(4), 913930. doi:10.1007/s11187-013-9503-y

Colle, R. (2011). $12^{\circ}$ - El análisis de contenido de las Comunicaciones, 2. Técnicas de análisis. España: Sociedad Latina de Comunicación Social, SLCS. Recuperado de: http:// www.revistalatinacs.org/067/ cuadernos/12_Colle_interior.pdf

Fernández, F. (2002). El análisis de contenido como ayuda metodológica para la investigación. Revista de Ciencias Sociales (Cr), 2(96). Recuperado de: https:// www.revistacienciassociales.ucr.ac.cr/images/ revistas/RCS96/03.pdf

Freixa i Baqué, E. (2003). ¿Qué es conducta? International Journal of Clinical and Health Psychology, 3 (3), 595-613. Recuperado de: http:// 
www.aepc.es/ijchp/articulos pdf/ijchp-89.pdf

Gartner, W. B. (1988). "Who Is an Entrepreneur?” Is the Wrong Question. American Journal of Small Business, 12(4), 11-32. doi: $10.1177 / 104225878801200401$

Glasziou, P., Irwig, L., Bain, C., \& Colditz, G. (2001). Systematic reviews in health care: a practical guide. Cambridge University Press. doi:/10.1017/CBO9780511543500

González, I. F., Urrútia, G., \& Alonso-Coello, P. (2011). Revisiones sistemáticas y metaanálisis: bases conceptuales e interpretación. Revista española de cardiología, 64(8), 688-696. doi:10.1016/j.recesp.2011.03.029

Gorgievski, M. J., \& Stephan, U. (2016). Advancing the psychology of entrepreneurship: A review of the psychological literature and an introduction. Applied Psychology, 65(3), 437-468. doi:10.1111/apps.12073

Ireland, D. \& Webb, J. (2007). A cross-disciplinary exploration of entrepreneurship research. Journal of management, 33(6), 891-927. doi:/10.1177\%2F0149206307307643

Kerlinger, F.N. (1988). Investigación del comportamiento. Técnicas y metodología. México: Editorial Interamericana.

Low, M., \& MacMillan, I. (2007). Entrepreneurship: investigación en el pasado y retos para el futuro. En Á. Cuervo, D. Ribeiro \& S. Roig, Entrepreneurship: conceptos, teoría y perspectiva (pp. 118-141). Valencia: Fundación Bancaja.

Lozano, J. M. (2009). De patos, gansos y cisnes: Revisiones narrativas, revisiones sistemáticas y meta -análisis de la literatura. Acta Médica Colombiana, 34(2S), 208-210. Recuperado de http:// www.actamedicacolombiana.com/ojs/ index.php/actamed/article/view/1705

Manchado Garabito, R., Tamames Gómez, S., López González, M., Mohedano Macías, L., D’Agostino, M. \& Veiga de Cabo, J. (2009). Revisiones sistemáticas exploratorias. Medicina y seguridad del trabajo, 55(216), 12-19. Recuperado de: http://scielo.isciii.es/pdf/mesetra/v55n216/ especial.pdf

Minniti, M. (2012). El emprendimiento y el crecimiento económico de las naciones. Economía industrial, 383, 23-30. Recuperado de: https:// www.mincotur.gob.es/Publicaciones/ Publicacionesperiodicas/EconomiaIndustrial/ RevistaEconomiaIndustrial/383/Mar\%C3\% ADa\%20Minnit.pdf

Muñoz-Justicia, J. \& Sahagún-Padilla, M. (2017). Ha- cer análisis cualitativo con Atlas.ti 7, Manual de uso. doi:/10.5281/zenodo.273997

Nagarajan, K. (2011). A History of Entrepreneurship: Review. International Journal of Business and Social Science, 2(9), 241-242. doi: $10.4324 / 9780203877326$

Poth, C., \& Ross, S. (2009). Meta-analysis, systematic review, or scoping review? Comparing methodologies in educational research. In Annual Conference of the Canadian Society for the Study of Education, Ottawa, ON, Canada.

Rodríguez, A. (2009). Nuevas perspectivas para entender el emprendimiento empresarial. Pensamiento Y Gestión, (26), 94-119. Recuperado de: http://rcientificas.uninorte.edu.co/ index.php/pensamiento/article/viewFile/864/508

Rosero, O., \& Molina, S. (2008). Sobre la investigación en emprendimiento. Informes Psicológicos, (10), 29-39. Recuperado de: http:// pepsic.bvsalud.org/pdf/ipsi/n10/n10a03.pdf

Santos, F., \& Barroso, M., \& Guzmán, C. (2013). La economía global y los emprendimientos sociales. Revista de Economía Mundial, (35), 177196. Recuperado de: https://www.sem-wes.org/ sites/default/files/revistas/rem35_10.pdf

Sassmannshausen, S. P., \& Volkmann, C. (2013). A bibliometric based review on social entrepreneurship and its establishment as a field of research (No. 2013-003). Schumpeter Discussion Papers. Recuperado de: https:// citeseerx.ist.psu.edu/viewdoc/download? doi $=10.1 .1 .472 .427 \&$ rep $=$ rep $1 \&$ type $=p d f$

Tangarife, A., Giraldo, D., \& Sanín, J. (2015). Globalización, Emprendimiento e Innovación en la Ciudad de Medellín: un dispositivo para la construcción de sujetos en el marco de la ciudad capitalista. En VIII Congreso Latinoamericano de Ciencia Política. Lima. Recuperado a partir de http://files.pucp.edu.pe/sistema-ponencias/ wp-content/uploads/2014/12/PanelALACIP.pdf

Veciana, J. (2007). Creación de empresas como programa de investigación científica. En Á. Cuervo, D. Ribeiro \& S. Roig, Entrepreneurship: conceptos, teoría y perspectiva (pp. 24-65). Valencia: Fundación Bancaja.

Virgen Ortiz, V., \& Cobo Oliveros, C., \& Betancourt Guerrero, B. (2014). Análisis bibliométrico del campo de formación de emprendedores. Cuadernos de Administración, 30 (52), 4453. Recuperado de: http://www.scielo.org.co/ pdf/cuadm/v30n52/v30n52a05.pdf 
Referencias citadas en el análisis de contenido deductivo:

Bandura, A., \& Locke, E. A. (2003). Negative selfefficacy and goal effects revisited. Journal of Applied Psychology, 88(1), 87-99. doi:10.1037/0021-9010.88.1.87

Cromie, S. (1987). Motivations of aspiring male and female entrepreneurs. Journal of Organizational Behavior, 8(3), 251-261. doi:10.1002/ job.4030080306

Davidsson, P., \& Wiklund, J. (1997). Values, beliefs and regional variations in new firm formation rates. Journal of Economic Psychology, 18(23), 179-199. doi:10.1016/S0167-4870(97) 00004-4

Fligstein, N. (1997). Social skill and institutional theory. American Behavioral Scientist, 40(4), 397405. doi: $10.1177 / 0002764297040004003$

Hisrich, R. D. (1990). Entrepreneurship/ intrapreneurship. American Psychologist, 45 (2), 209-222. doi:10.1037/0003066X.45.2.209

Johannisson, B. (1988). Business formation - a network approach. Scandinavian Journal of Management, 4(3-4), 83-99. doi:10.1016/09565221(88)90002-4

Krauss, S. I., Frese, M., Friedrich, C., \& Unger, J. M. (2005). Entrepreneurial orientation: A psychological model of success among southern african small business owners. European Journal of Work and Organizational Psychology, 14 (3), 315-344. doi: $10.1080 / 13594320500170227$

Lee, J. (1999). Retail niche domination among african american, jewish, and korean entrepreneurs: Competition, coethnic advantage and disadvantage. American Behavioral Scientist, 42 (9), 1398-1416. doi:10.1177/00027649921954967

Morris, M., Schindehutte, M., \& Allen, J. (2005). The entrepreneur's business model: Toward a unified perspective. Journal of Business Research, 58(6), 726-735. doi:10.1016/ j.jbusres.2003.11.001

Obschonka, M., Schmitt-Rodermund, E., Silbereisen, R. K., Gosling, S. D., \& Potter, J. (2013). The regional distribution and correlates of an entrepreneurship-prone personality profile in the united states, germany, and the United Kingdom: A socioecological perspective. Journal of Personality and Social Psychology, 105(1), 104-122. doi:10.1037/a0032275

Parasuraman, S., Purohit, Y. S., Godshalk, V. M., \&
Beutell, N. J. (1996). Work and family variables, entrepreneurial career success, and psychological well-being. Journal of Vocational Behavior, 48(3), 275-300. doi:10.1006/ jvbe. 1996.0025

Raposo, M., \& do Paço, A. (2011). Entrepreneurship education: Relationship between education and entrepreneurial activity. [Educación emprendedora: Relación entre educación y actividad emprendedora] Psicothema, 23(3), 453 -457. Retrieved from http:// www.psicothema.com/pdf/3909.pdf

Rauch, A., \& Frese, M. (2007). Let's put the person back into entrepreneurship research: A metaanalysis on the relationship between business owners' personality traits, business creation, and success. European Journal of Work and Organizational Psychology, 16(4), 353-385. doi:10.1080/13594320701595438

Rothenberg, S. (2007). Environmental managers as institutional entrepreneurs: The influence of institutional and technical pressures on waste management. Journal of Business Research, 60(7), 749-757. doi:10.1016/ j.jbusres.2007.02.017

Schmitt-Rodermund, E. (2004). Pathways to successful entrepreneurship: Parenting, personality, early entrepreneurial competence, and interests. Journal of Vocational Behavior, 65(3), 498518. doi:10.1016/j.jvb.2003.10.007

Verheul, I., Thurik, R., Grilo, I., \& Van der Zwan, P. (2012). Explaining preferences and actual involvement in self-employment: Gender and the entrepreneurial personality. Journal of Economic Psychology, 33(2), 325-341. doi:10.1016/j.joep.2011.02.009

Weiler, S., \& Bernasek, A. (2001). Dodging the glass ceiling? networks and the new wave of women entrepreneurs. Social Science Journal, 38 (1), 85-103. doi:10.1016/S0362-3319(00) 00111-7 